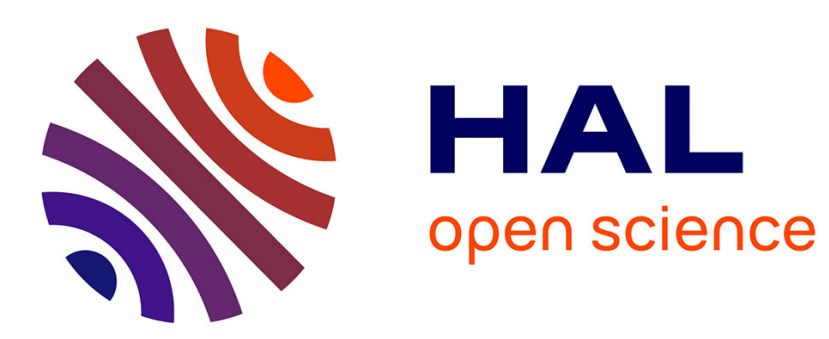

\title{
Réforme de la fonction publique et réorganisation de l'état
}

Gilles Jeannot

\section{To cite this version:}

Gilles Jeannot. Réforme de la fonction publique et réorganisation de l'état. Revue Esprit, 2008, 350, pp.94-109. hal-00583185

\section{HAL Id: hal-00583185 \\ https://hal-enpc.archives-ouvertes.fr/hal-00583185}

Submitted on 11 Jul 2013

HAL is a multi-disciplinary open access archive for the deposit and dissemination of scientific research documents, whether they are published or not. The documents may come from teaching and research institutions in France or abroad, or from public or private research centers.
L'archive ouverte pluridisciplinaire HAL, est destinée au dépôt et à la diffusion de documents scientifiques de niveau recherche, publiés ou non, émanant des établissements d'enseignement et de recherche français ou étrangers, des laboratoires publics ou privés. 


\title{
Réforme de la fonction publique et réorganisation de l'Etat
}

\author{
Gilles Jeannot
}

Esprit, décembre 2008, p. 94- 109

Après le livre blanc sur l'avenir de la fonction publique remis par Jean-Ludovic Silicani ${ }^{1}$, et un projet de loi sur la mobilité ${ }^{2}$, les grandes lignes de la réforme de la fonction publique de l'Etat commencent à se dessiner. Cela ne signifie pas que les orientations soient totalement claires. La loi organique sur les lois de finances (Lolf) votée par l'ensemble des partis au parlement en 2006, était construite autour de quelques principes simples (clarification des programmes pour le parlement, indicateurs de résultats, autonomie de gestion) et elle trouvait une origine unifiée dans la "gestion par programme" clairement issue de ce que l'on a appelé le "New public management". Les propositions sur la fonction publique, par contraste, associent des propositions diverses et pas nécessairement cohérentes et vont chercher leurs modèles dans des lieux très différents.

Les notions de "mérite", de "valeurs" ou de "métiers" censées donner sens à la démarche sont toutes passablement ambiguës. Et s'attacher simplement au droit n'est pas nécessairement plus éclairant, dans la mesure où, dans ce domaine plus encore que dans d'autres, l'écart est énorme entre ce que permet le droit et ce qui est effectivement utilisé ${ }^{3}$. Il est utile alors de mettre en évidence les modèles auxquels empruntent les diverses propositions faites, pour en dégager la philosophie, mais surtout pour appréhender les effets possibles des orientations prises en les rapportant à des expériences connues à l'étranger ou en France.

\section{La « prime au mérite » et les limites du modèle du new public management}

L'attribution de «primes au mérite » est sûrement le volet le plus débattu des nouvelles orientations, il n'en est pas pour autant nécessairement le plus déterminant. Derrière le terme ambigu de mérite se cache l'idée de primes liées à la performance du service. Une telle idée était promue pour diverses raisons. En premier lieu, elle constitue un des thèmes dominants des discours présidentiels ${ }^{4}$ et une de ses premières mises en œuvre dans le gouvernement

\footnotetext{
${ }^{1}$ Livre blanc sur l'avenir de la fonction publique. Faire des services publics et de la fonction publique des atouts pour la France, Ministère du budget des comptes publics et de la fonction publique, avril 2008, $236 \mathrm{p}$.

${ }^{2}$ Loi sur la mobilité et les parcours professionnels dans la fonction publique, soumise en première lecture au Sénat le 29 avril 2008.

${ }^{3}$ Rappelons par exemple que le droit de licenciement pour insuffisance professionnelle existe déjà dans la fonction publique.

4 "Le mérite et l'effort doivent jouer une part beaucoup plus importante dans la détermination des rémunérations des agents publics. Il faut réduire la part automatique et égalitaire au profit d'augmentations méritées, qui traduisent une reconnaissance des efforts et du mérite de chacun" Sarkozi Nicolas, Vœux aux fonctionnaires, Lille, 11 janvier 2008. Cette manière de relier prime et augmentation se rapproche d'une pratique qui s'est diffusée dans le secteur privé. Dans le secteur privé, la question a été traitée en particulier par l'instauration croissante d'augmentations individualisée en complément des augmentations collectives, celles-ci passent de 25 $\%$ à $35 \%$ entre 1992 et 1998 pour les cadres et de $9 \%$ à $19 \%$ pour les non cadres. Cette tendance doit cependant être relativisées : seules 4, $6 \%$ des entreprises n'utilisent que des augmentations individualisées, et
} 
précédent avait été au ministère de l'intérieur. En second lieu, une telle proposition vient tout naturellement compléter la loi organique sur les lois de finances (LOLF). En effet, puisque la nouvelle nomenclature budgétaire construit l'intervention publique autour de la mesure d'indicateurs de résultats, pourquoi ne pas appliquer le même principe aux fonctionnaires qui seraient ainsi incités à atteindre ces résultats?

Pour les cadres dirigeants, l'application stricte d'un raisonnement économique au comportement des fonctionnaires semble pouvoir être rattachée au modèle du "Public choice". Pour les auteurs qui appliquent ${ }^{5}$ le raisonnement économique dans l'analyse de la démocratie et de la bureaucratie, les responsables hiérarchiques dans les bureaucraties gonflent leur importance en augmentant la taille de leur service et leur budget et non en faisant des économies. Il s'agit alors d'attribuer des missions claires à ces responsables et de relier une part essentielle de leurs salaires à leur capacité à atteindre les objectifs fixés. Ce raisonnement a orienté la création des agences et leur conduite en Grande Bretagne. Quels en sont les effets ? Si l'agence produit un service défini par un seul critère de qualité ou de prix, le mécanisme peut être efficace. Si elle a en charge un bien public dont la protection inclut des arbitrages entre objectifs contradictoires, une telle orientation peut être contreproductive. Il n'est pas sûr, cependant, que la mise en place de primes de performance pour les cadres dirigeants dans la fonction publique d'Etat en France ait vraiment été envisagée en ces termes. Dans bien des cas, pour ceux-ci, l'introduction de la dimension de «mérite » a été tout autant une manière de légitimer un rétablissement d'une hiérarchie salariale qui avait été progressivement été érodée ${ }^{6}$.

Pour ce qui concerne les autres agents, les promoteurs les plus nuancés de la mesure la conçoivent, dans la lignée du New public management, moins comme une pure incitation économique que comme un moyen pédagogique permettant de mettre en évidence la contribution de chacun à l'efficacité de l'action publique ${ }^{7}$. Cependant ce petit plus pourrait bien devenir un grand moins. Trois problèmes se cumulent. Tout d'abord la difficulté de définir des indicateurs de performance pertinents, qui apparaît dans les premières évaluations de la Loi organique sur les lois de finances ${ }^{8}$. Ensuite, une fois le critère pertinent trouvé, si la chose est possible, il faut encore attribuer la performance à un individu particulier. Dans bien des cas, l'efficacité est liée à l'action collective et la prime individuelle favorise ceux qui ne jouent pas le jeu. Enfin, le lien entre l'obtention de la prime et la motivation n'est pas aussi immédiat que ne le suppose une psychologie économique rudimentaire. La motivation économique se combine en effet de manière complexe avec une motivation intrinsèque liée à un certain sens du devoir à accomplir, à l'éthos du service public ${ }^{9}$ ou à l'intérêt porté au travail. Cette question est l'objet de nombreuses études de psychologie qui invalident largement l'hypothèse de réaction immédiate des comportements à l'incitation économique. Par exemple, le fait de payer une somme modique pour le don de sang éloigne certains donneurs qui contribuaient volontiers à titre gratuit mais qui considèrent que la somme

\footnotetext{
récemment un retour vers les augmentations partagées semble se dessiner. De Bayer Anne, Georgeault Valérie, Maréchal Pierre, La rémunération au mérite, mode ou nécessité ? Les Cahiers du groupe Bernard Bruhnes, nov. 2004, $\mathrm{n}^{\circ}$ 13. Barreau Jocelyne, Brochard Delphine, Les politiques de rémunération des entreprises : écarts entre pratiques et discours, Travail et emploi, $\mathrm{n}^{\circ}$ 93, janvier 2003

${ }^{5}$ Niskannen William, Bureaucracy and representative government, Aldine Atherton Chicago, 1971.

${ }^{6} \mathrm{Ce}$ sujet a été l'objet d'un autre rapport de Jean-Ludovic Silicani: Rapport sur la rémunération des directeurs d'administration centrale, avril 2004.

7 DGAFP, GRH et LOLF, Gestion et reconnaissance de la performance, DGAFP, juillet 2005, 87 p., Trosa Sylvie, "payer les fonctionnaires au mérite ?", Sociétal, n 44, 2004.

${ }^{8}$ Par exemple Matelly J-H, Mouhanna C; Police, des chiffres et des doutes, Michallon, 2007.

${ }^{9}$ Perret Bernard, "Les limites annoncées de la politique économique", Esprit, ${ }^{\circ}{ }^{\circ}, 2007$, p 88-97 (p. 96).
} 
allouée est trop faible pour rémunérer leur don. Ainsi, certains agents qui obtiennent un complément somme toute limité de salaire relativisent leur motivation liée au sens du service public et d'autres agents qui ne «méritent» pas la prime trouvent une bonne raison pour en faire encore moins. Tout cela est bien compris des cadres intermédiaires en charge de l'attribution des primes qui, sagement, vont répartir également la prime ou l'attribuer par roulement si les règles ne permettent pas une répartition égalitaire, comme on a pu l'observer dans les collectivités territoriales ou dans le privé ${ }^{10}$.

Cette manière de mettre en avant l'incitation économique immédiate des agents n'occupe plus une position aussi centrale qu'à l'origine du courant de réforme déployé dans les pays anglosaxons. Les pays comme l'Australie qui avaient mis en place des critères objectifs de détermination de telles primes sont revenus en arrière ${ }^{11}$. Par ailleurs, après 2000 , le mouvement de la gestion des compétences «competency management » qui se généralise dans les pays marqués par le New Public Management repose sur une caractérisation des qualités des individus et implicitement sort du modèle de gestion des ressources humaines fondé sur des incitations économiques immédiates. Et d'ailleurs le livre blanc propose des niveaux relativement faibles de 5 à $7 \%$ pour les premiers niveaux de grades en combinant prime collective et prime individuelle ${ }^{12}$.

\section{Une fonction publique de métier, le changement dans la continuité}

A cet affichage un peu provocateur d'un modèle anglo-saxon, on peut ensuite opposer, dans un bon nombre des propositions faites, la simple poursuite d'une tradition de réforme de la fonction publique d'Etat en France au nom du «métier ».

Le terme de «métier» n'est pas moins ambigu que celui de «mérite ». En effet, ce terme évoque aussi bien les corporations de métiers de l'ancien régime (dont on trouve l'équivalent contemporain dans les corps d'Etat) que la reconnaissance du savoir faire des individus. Le métier comme corporatisme est souvent mis en cause, alors qu'il est mis en valeur comme professionnalisme. Le rapport public du conseil d'Etat de 2003 peut ainsi justement réordonner les réformes du passé autour d'un "principe directeur simple : l'organisation de la fonction publique doit permettre dans le respect des valeurs fondatrices et des principes de base de la fonction publique, l'adaptation des ressources humaines aux missions de service public et aux métiers et qualifications professionnelles qu'elles impliquent"13. Le bilan de cette réforme incrémentale a été fait à de nombreuses reprises. Elle a pu donner naissance à des actions concrètes locales qui témoignent d'une souplesse de la fonction publique plus grande qu'on ne le pense souvent ${ }^{14}$. Cela permet par exemple dans certains ministères (Equipement, police) une différenciation réelle des carrières. Cependant ces injonctions ont été interprétées de manière très différentes selon les ministères et ces innovations n'ont jamais

\footnotetext{
${ }^{10}$ Ce mécanisme a été en particulier décrit dans le cas des collectivités locales où seuls les agents les plus manifestement mis hors du travail sont privés de la prime par Crozet P ; Desmarais C. , 2005, l'encadrement des orgnisations publiques face à la modernisation managériale, une position intenable ? actes du XVIe congrès de l'AGRH, septembre. Des comportements comparables dont observés dans le privé. Delavallée Eric, "La vraie fonction de la rémnération au mérite", L'expansion management review, mars 2003. Une enquête Cegos de 2003 révèle aussi que $65 \%$ des cadres du privé ne considèrent pas ces mécanismes "adaptés et mobilisateurs".

${ }^{11}$ Pilichowski E (2005), Evolution des politiques de gestion des ressources humaines dans les pays de l'OCDE : une analyse des résultats de l'enquête de l'OCDE sur la gestion stratégique des ressources humaines.

${ }^{12}$ Ce qui fait pour un salaire moyen de 1973 euros mensuel en catégorie C et en affectant la moitié de la prime au mérite au collectif, une prime individuelle moyenne de 50 euros par mois.

${ }^{13}$ Conseil d'Etat, 2004, p. 302.

${ }^{14}$ Pallez Frédérique, «Les souplesses cachées du mammouth », Le journal de l'Ecole de Paris, janvier 1999, p.3.
} 
suffit à éradiquer les tendances bureaucratiques de la gestion et la prédominances de règles formelles sur une véritable différenciation des qualités professionnelles des agents.

Le rapport Silicani reprend le principe d'une reconnaissance des qualités professionnelles dans un ensemble de mesures concernant le recrutement et les parcours des individus. Si le principe du concours pour l'entrée dans la fonction publique, entendu d'abord comme garantie d'accès de tous, n'est pas remis en cause, le rapport propose de renforcer la part du jugement sur les qualités professionnelles dans différents domaines. Les concours externes et internes devront être très largement conduits sur des épreuves professionnelles ${ }^{15}$. Par ailleurs, pour la mobilité et l'accès au poste, le rapport propose que ce soit le chef de service qui recrute parmi les différents postulants à un poste. L'annonce par Eric Woerth le 24 septembre 2008 de la suppression du classement de sortie de l'école nationale d'administration qui permettait aux individus les mieux classés de choisir les corps les plus prestigieux a été présentée comme un pas dans cette direction. Cependant la responsabilisation des chefs de services serait bien limitée si dans le même temps ces "grands corps" n'étaient pas réformés et si ce premier recrutement valait pour toute la carrière. Enfin le rapport préconise de concentrer le jugement évaluatif sur la promotion de grade et d'en faire l'occasion d'un jugement sur le contenu des postes à occuper ou déjà occupés ("Les agents ayant occupé pendant une certaine durée (trois ans par exemple) un ou plusieurs postes comportant des responsabilités ou des sujétions professionnelles particulièrement lourdes, pourraient bénéficier d'un avancement de grade accéléré"16).

La volonté de renforcer les qualités professionnelles des agents se retrouve aussi dans un ensemble de textes promouvant la formation et l'expérience ${ }^{17}$ qui introduisent la reconnaissance d'un droit individuel de formation et des modalités de « reconnaissance des acquis de l'expérience professionnelle en matière de recrutement et de promotion". Ces propositions permettent de transcrire des textes s'appliquant au privé, tout comme de répondre à certaines contraintes règlementaires européennes. Cette évolution des textes devra toutefois, pour être jugée, être rapportée aux moyens supplémentaires mis pour rendre effectifs ces droits.

Cependant, la principale nouveauté du rapport est l'introduction du poste comme référence pour la détermination d'une part du salaire. "La rémunération de chaque agent titulaire de la fonction publique comprendrait deux composantes, l'une liée au grade, et l'autre à l'emploi se substituant à l'ensemble des éléments actuels de rémunération" ${ }^{18}$. Cette logique s'était progressivement insinuée dans la fonction publique d'Etat. C'était le cas en particulier de la nouvelle bonification indiciaire (NBI) qui ajoute des points d'indices à certains postes d'encadrement ou forte sujétion ou des "emplois fonctionnels", règles salariales spécifiques pour certains postes de direction.

Mettre ainsi en avant le poste est une manière d'aller chercher des références à un certain secteur privé. Dans le modèle de la fonction publique, pour simplifier, le salaire est déterminé par la réussite d'un concours qui ouvrirait un droit à la carrière où les échelons sont "gravis" à l'ancienneté, ce droit allant même après la retraite, la pension relevant plus en droit du salaire

\footnotetext{
${ }^{15}$ Ce sujet a fait l'objet d'un rapport Desforges/Chalvron remis le 19 fev 2008 au gouvernement.

${ }^{16}$ p. 126

${ }^{17}$ Loi n ${ }^{\circ}$ 2007-148 du 2 février 2007 de modernisation de la fonction publique, Décret 2007-1470 du 15 octobre 2007 relatif à la formation professionnelle tout au long de la vie.

${ }^{18}$ La nouveauté doit être relativisée, ce thème ayant été évoqué dans un comité interministériel du 15 novembre 2001 (Conseil d'Etat, 2004, p. 360-361).
} 
différé. C'est bien sûr une caricature puisque la promotion ne s'effectue pas à l'ancienneté dans toutes les administrations et que les passages d'un corps à un autre sont loin d'être marginaux $^{19}$. A cette caricature en répond une autre représentant le salaire dans le privé attribué sur la seule appréciation de la "performance" de l'individu. La définition du salaire dans le privé est en fait prise entre plusieurs modèles concurrents. Le salaire peut reposer sur la mesure de ce qui est produit - le salaire aux pièces dans le travail à domicile — sur ce qui est vendu - la guelte pour les vendeuses des magasins ou la commission à la vente qui vient s'ajouter à un fixe faible des VRP. A cela s'oppose cependant un modèle qui repose sur une caractérisation de la complexité des postes et des prescriptions associées et sur le niveau scolaire permettant de les accomplir, ce que l'on appelle la "qualification" Ce modèle de la qualification s'est en particulier imposé après la guerre dans l'industrie avec les grilles Parodi. Un tel modèle, appliqué de manière relativement rigide, a servi de base à la classification des emplois à France télécom et à La poste ${ }^{20}$. Il a été aussi appliqué dans un certain nombre de fonctions publiques d'autres pays. Il a l'avantage de permettre une discussion sur l'appréciation des postes qui soit détachée des individus qui les occupe ${ }^{21}$. Une étude comparative des effets de différentes manières de reconnaître les qualités professionnelles des fonctionnaires note d'ailleurs que celles qui reposent sur une caractérisation des postes de travail sont celles qui ont les impacts pratiques les plus marqués ${ }^{22}$.

La principale incertitude actuelle concerne les modalités de basculement du régime actuel des primes au nouveau régime fondé sur le poste. La rémunération fonctionnelle viendrait se substituer en partie aux primes (régime indemnitaire) : "il est proposé d'accroître progressivement la part moyenne de la rémunération fonctionnelle en passant, en dix ans d'environ $15 \%$ (niveau actuel du régime indemnitaire par rapport au total des rémunérations) à $25 \% " 23$. Or s'il est difficile d'expliquer les différences de régimes indemnitaires entre les ministères avec en tête le ministère des finances, au milieu les ministères techniques et en fin de course l'éducation nationale (proximité de la décision, réponse à une concurrence sur certains emplois technique...), il apparaît difficile de soutenir que ce régime reflète en l'état une échelle de complexité ou de difficulté des fonctions. Et même si le rapport prévoit qu'une telle réforme a un coût ${ }^{24}$, il ne donne pas les conditions d'affectation de ce budget. Ne risquet-on pas alors de "découvrir", pour raisons d'économie, que les postes au ministère des finances sont par nature plus complexes que ceux de l'éducation nationale, et de manière générale que la hiérarchie du régime indemnitaire actuel correspond à une hiérarchie des postes occupés et de décrédibiliser par là la démarche?

\footnotetext{
${ }^{19}$ Comme le montre une étude originale reposant sur le suivi de cohortes d'individus à partir du fichier de paie, $70 \%$ des agents de catégorie B entrés en 1983 sont passés en catégorie A 23 ans plus tard et $41 \%$ des agents entrés en 1997 en B sont passés en A 8 ans plus tard. Ce phénomène notable (hors promotion de corps entiers comme les instituteurs) a sûrement combiné les effets de surqualification avec les possibilités offertes par l'évolution globale de la qualification des fonctionnaires de l'Etat et s'explique en partie par le fait que des agents de catégorie B ont passé et réussi les concours externes et pas seulement internes. Il témoigne cependant d'une adaptation du corps social de la fonction publique. Bessière Sabine, Pouget Julien, "Les carrières dans la fonction publique de l'Etat, premiers éléments de caractérisation" in: Les salaires en France, INSEE, 2007, p. 5166.

${ }^{20}$ Alter Norbert, L'innovation ordinaire, Paris, PUF, 2001

${ }^{21}$ Certains soulignent que parfois "la personne fait le poste", cependant cet argument des promoteurs du modèle français de la compétence est plus proche d'un ajustement du modèle de la qualification des postes que du modèle d'un jugement porté sur la seule personne. Oiry E., De la qualification à la compétence, rupture ou continuité, L'harmattan, 2003.

${ }^{22}$ Hood C. Lodge M., "Competency, Bureaucracy and Public Management Reform: a comparative Analysis, Governance, vol 17, $\mathrm{n}^{\circ}$ 3, juillet 2004, p. 313-333.

${ }^{23}$ Livre blanc... p. 132.

${ }^{24}$ Le chiffre de 1 milliard d'euros a été avancé.
} 


\section{La fin des corps et le modèle de la fonction publique territoriale}

A côté de ces références au secteur privé, la fonction publique territoriale apparaît pour certains comme le modèle le plus crédible, par sa proximité, pour orienter la réforme de l'Etat. La référence à ce modèle apparaît explicitement dans l'idée de «cadres statutaires », qui viendraient se substituer aux corps, promue par le rapport du Conseil d'Etat de $2003^{25}$ et reprise dans le récent livre blanc. L'idée est de remplacer les corps actuels par des "cadres d'emplois" organisés dans une grille simple croisant d'une part des filières thématiques (administration générale, financière et fiscale, sociale, éducation et recherche, culturelle, technique, sécurité) et d'autre part cinq niveaux (exécution, maîtrise, cadres de premier niveau, cadre de second niveau, direction). Le cinquième niveau ne correspondrait pas à un corps d'appartenance mais à l'occupation effective d'un poste de direction (ce qui est une autre manière de retrouver la logique de qualification énoncée précédemment). Le tableau de principe ainsi défini est de 35 cases et le rapport du Conseil d'Etat envisageait de superposer les cadres d'emplois aux corps existants. Le livre blanc actuel propose plutôt une accélération de la fusion des corps pour converger progressivement vers une cinquantaine de cadres d'emplois. En tous les cas, cependant, ce modèle conduit à une réduction nette par rapport aux sept cent corps actuels et transforme de fait la nature de ces corps.

Cette réforme est justifiée, selon ses promoteurs, par la volonté de faciliter certaines mobilités interministérielles. L'argument vaut en particulier pour les fonctions administratives transversales. Les responsabilité administratives sont en effet parfois occupées de manière relativement temporaire et en "amateur" par des agents de corps divers. Ce sujet avait en particulier été au centre des discussions qui ont accompagné la rédaction d'un répertoire interministériel des métiers. L'exemple régulièrement évoqué alors, très proche de beaucoup des participants des groupes de travail, était celui de la fonction de responsable des ressources humaines. Un professionnel des ressources humaines circulant plus facilement, du fait de ce nouveau cadre, entre des petits ministères, pourrait approfondir son professionnalisme tout en facilitant la circulation des expériences entre ces administrations. Mais cette manière de relier l'ouverture du cadre à l'enrichissement professionnel est moins convaincante pour les filières pour lesquelles le nouveau cadre correspond très largement aux frontières de ministères et de corps existants comme l'éducation ou de la sécurité. Et il en va de même pour les agents des grands ministères qui offrent déjà des possibilités importantes de mobilité.

Le décalage entre le problème traité et la révolution que représente la mise en cause du système des corps est frappant, il faut donc aller au-delà de cet effet immédiat pour justifier un tel projet. Cette remise en cause des corps doit alors être comprise dans une stratégie de déplacement des rapports de forces entre directions et syndicats. Le rapport de Marcel Pochard repose sur un raisonnement sociologique. S'il ne nie pas que le statut de la fonction publique est en droit très souple et permet de prendre en compte la reconnaissance des qualités professionnelles des agents et que celle-ci est d'ailleurs dans l'esprit même du statut, il constate cependant que le statut génère les conditions sociales de son détournement. "Si le statut n'interdit pas (...) les formes de gestion vertueuses, il n'empêche pas non plus les autres, et on peut se demander au contraire s'il ne les encourage pas peu ou prou" ${ }^{26}$. Le fait que les

\footnotetext{
${ }^{25}$ Conseil d'Etat, Rapport public 2003. Perspectives pour la fonction publique, Etudes et documents du Conseil d'Etat, $n^{\circ}$ 54, La Documentation française, 2004, 446 p.

${ }^{26}$ Ibid p. 263.
} 
décisions soient toutes prises par corps a en particulier, selon ce rapport, soutenu un syndicalisme organisé autour de la défense de chacun des corps. Cela se traduit alors par un accord dans les commissions administratives paritaires (CAP) qui rassemble direction et syndicats pour privilégier les membres du corps dans la promotion de grade sur ceux qui viennent d'arriver en détachement et qui doivent attendre leur tour ${ }^{27}$. Le rapport Pochard proposait alors de changer les règles du jeu par exemple en proposant des CAP intercorps qui déstabiliseraient de tels mécanismes.

La référence au modèle de la fonction publique territoriale peut aussi être lue en creux dans deux thèmes secondaires du livre blanc, l'insistance du rapport sur la question des «stagiaires » et la multiplication des emplois fonctionnels aux plus hauts niveaux de la hiérarchie. Le recrutement dans la fonction publique d'Etat n'est définitif qu'après une période de stage (souvent d'un an) et avis positif donné de manière quasi automatique par le responsable hiérarchique. En premier lieu, le livre blanc propose de mettre en cause la pratique d'attribution systématique du statut de fonctionnaire aux stagiaires et d'en faire une véritable période d'essai. Cette manière de vouloir rendre les services juges de l'embauche définitive de la personne qui a réussi le concours n'est pas sans rappeler le cas de figure des "reçus-collés" propre à la fonction publique territoriale. En effet, dans cette fonction publique, le lauréat d'un concours doit être recruté sur entretien par une des collectivités territoriales, faute de cela, il perdra le bénéfice du concours. En second lieu, les statuts spécifiques pour les emplois de direction permettent à la fois le recrutement et la révocation discrétionnaire et pourraient permettre de soutenir une tendance à la politisation des recrutements. Comme le note Bernard Perrin ${ }^{28}$, ce spoil system à la française n'a cessé de se renforcer ces dernières années dans les collectivités locales et surtout de concerner progressivement des échelons plus bas de la hiérarchie.

\section{Contrats de droit privé, intérim et modèles européens}

Les longs développements du livre blanc sur la question des contractuels et finalement la proposition d'introduire un contrat de droit privé dans la fonction publique d'Etat ne peut être comprise indépendamment de la référence à des modèles européens aux deux sens du terme (de la Commission européenne et de pays européens particuliers).

L'idée qui est débattue ici est de réserver un statut spécifique aux agents à ceux qui effectuent des missions spécifiques. Elle a été introduite en particulier par Gérard Longuet dans un rapport de 1979 qui est considéré comme le point de vue libéral sur la fonction publique ${ }^{29}$. Dans cette perspective, l'agent qui instruit un dossier administratif et qui peut éventuellement être soumis à des pressions des pétitionnaires doit avoir un statut qui le protège de toute influence. Mais l'agent qui effectue des prestations de service comme le professeur pourrait avoir un statut moins protecteur. C'est en partie s'inspirer du modèle allemand qui distingue les Beamte, les fonctionnaires en charge des fonctions régaliennes, et les Angestelte, les

\footnotetext{
27 ibid p. 276.

${ }^{28}$ Perrin Bernard, "Les emplois de direction dans les collectivités territoriales : la capacité d'adaptation, vertu première", Revue Française d'administration publique, n 116, 2005, pp. 583-594.

${ }^{29}$ Rouban Luc, La fonction publique, La découverte, 1996.
} 
agents accomplissant d'autres tâches et qui n'ont pas besoin des mêmes garanties d'indépendance.

Ce serpent de mer des débats sur la fonction publique est réapparu avec la mise œuvre de la politique de libre circulation des personnes portée par la commission européenne. Le texte européen suit le même principe que le modèle allemand et ne laisse les Etats libres de réserver l'accès à la fonction publique à leurs ressortissants que pour les fonctions régaliennes. La distinction a été, depuis, affinée dans une jurisprudence où l'on retrouve la même casuistique que dans les décisions concernant l'application du droit de la concurrence au service public. Cette jurisprudence porte sur le cas des capitaines de bateaux qui se voient confier des missions régaliennes d'état civil, pour prendre en compte par exemple une naissance ou un décès à bord. La cour reconnaît alors la spécificité pour les marins au long cours mais pas pour ceux qui font du cabotage et qui pourront toujours faire régulariser la situation lors de leur arrivée au port en fin de journée (CJCE, 30/09/2003). L'ampleur du phénomène d'accès des européens à la fonction publique d'Etat française est des plus réduite, mais les conséquences de certaines jurisprudences peuvent être très importantes. Et de la même manière que les règles de concurrence qui ne visaient pas les entreprises publiques ont entraîné de fait la plus importante réforme du secteur public que nous ayons connu depuis ces dernières années, il est possible d'envisager que de la même manière indirecte, des jurisprudences concernant des cas isolés entraînent des effets en cascade. En l'occurrence la première jurisprudence (arrêt Bubeaud CJCE 9 sept. 2003) portait sur la réclamation d'une directrice d'un hôpital portugais qui s'était vu refuser l'accès à la direction d'un hôpital français au prétexte qu'elle n'avait pas suivi les cours de l'école nationale de la santé publique. La Cour a tranché en sa faveur. Une telle décision pose des problèmes tout à fait délicats. Tout d'abord comment concevoir un mode de sélection qui ne conduise pas à prendre tous les ressortissants européens et permette de les comparer à des candidats français qui passent un concours ? Cela a conduit à prévoir une nouvelle modalité d'accès aux concours existants incluant une référence à l'expérience, ce qui est une autre manière de diffuser le modèle professionnel. Ensuite, se pose la question d'une "discrimination à rebours". En effet, comment, alors que l'on accepte de prendre en compte l'expérience d'un directeur d'hôpital européen non français, refuser d'appliquer le même traitement à un directeur français de clinique privée ? En droit pur $^{30}$ la Cour a toujours considéré les Etats souverains en la matière mais certains proposent une lecture maximaliste des effets d'entraînement associés à cette jurisprudence qui conduiraient à faire des fonctionnaires "des travailleurs comme les autres"31. A ce modèle allemand s'est ajouté, depuis, l'exemple de la fonction publique italienne, ce pays ayant transféré en bloc ses fonctionnaires vers des contrats de droit privé. Le Livre blanc rejette l'exemple italien : ces contrats de droit privé, dans la mesure où ils doivent préserver certaines spécificités de la fonction publique ne sont que partiellement comparables aux contrats de droit commun. Il hésite ensuite devant le modèle allemand. En effet la transcription de ce principe rencontre une difficulté d'importance : le principal ministère générateur actuel de contrats dans la fonction publique est l'armée, fonction régalienne par excellence.

Finalement, après ce tour d'horizon européen, Le livre blanc opte pour une position qui consiste à maintenir la possibilité de contrats de droit public pour les fonctions régaliennes, ce qui est dans la continuité de l'existant et propose d'introduire progressivement des contrats de

\footnotetext{
${ }^{30}$ Lemoyne Deforges, J.-M., (2003). L'adaptation de la fonction publique française au droit communautaire : rapport au Ministre de la fonction publique, de la réforme de l'Etat et de l'aménagement du territoire. Paris, Dalloz.

${ }^{31}$ Fernandes Leal C., Onnée-Abbruciati M. L., Le fonctionnaire est-il un salarié comme les autres ? Pensions de retraite dans les fonctions publiques en Europe, Bruxelles, Bruylant, 2003.
} 
droit privé pour les fonctions non régaliennes. Cet usage semble limité à la résolution de problèmes particuliers (besoins occasionnels, carrières courtes, compétences rares, recrutement de personnes éloignées de l'emploi public (handicapés, personnes faiblement diplômées)), cependant il ouvre aussi un cas plus large "faciliter les échanges entre le secteur public et le secteur privé".

De manière plus immédiate la loi sur la mobilité et les parcours professionnels dans la fonction publique introduit un autre cas de figure qui est celui de l'intérim. La possibilité de l'intérim, présentée là aussi comme exceptionnelle, nous entraîne dans un autre pays de référence, la Grande Bretagne. Le gouvernement britannique a en effet largement laissé se développer des sociétés de placement spécialisées dans des métiers de la fonction publique comme les infirmières et les professeurs, créant une nouvelle figure de la dualisation de la fonction publique Cela a suscité le foisonnement d'agents spécialisés dans l'intérim des enseignants comme Teaching Personnel ou Timewise teachers. On estime à 20000 le nombre de "supply teachers" intervenant chaque jour dans les écoles, dont une partie vient des pays du Commonwealth ${ }^{32}$.

La tendance à la dualisation ${ }^{33}$ de la fonction publique sur le modèle allemand conforté par les réglementations européennes, sur le mode britannique de l'intérim ou de manière plus discrète par simple transfert de fonctions autrefois assurées par les administrations au secteur privé, est une tendance forte au niveau international. Cependant la ligne de partage entre les vrais fonctionnaires, assurant des fonctions régaliennes, et les autres ne va pas de soi comme en témoigne les hésitation du Livre blanc sur la question. On peut alors se demander si le partage régalien/non régalien est bien pertinent pour saisir les évolutions contemporaines de la plusvalue apportée par le travail des agents publics ${ }^{34}$.

\section{Plans de reconversion et modèle des entreprises publiques françaises}

Lorsqu'on passe des idées et projets rassemblés dans le livre blanc sur la fonction publique aux premières applications pratiques avec le projet de loi sur la mobilité et les parcours professionnels dans la fonction publique qui est passé en première lecture au Sénat le 29 avril 2008, c'est un autre modèle qui se profile de manière plus discrète : celui des entreprises publiques françaises. En effet, si la mobilité des fonctionnaires est une des voies classiques des réformes, certaines dispositions de la nouvelle loi, couplées à la révision générale des politiques publiques (RGPP), laissent entrevoir la possibilité de véritables plans de restructuration calqués sur ceux qui ont été engagés dans les entreprises publiques ouvertes à la concurrence et en particulier à La poste et à France télécom.

La question de la mobilité fonctionnelle des fonctionnaires dans la vie courante des services est un enjeu souvent plus rhétorique que réellement pratique. Le thème de la mobilité a l'avantage d'allier la modernité avec les fondements même du statut. La dénonciation des blocages juridiques ou des tracasseries bureaucratiques opposées à la mobilité est forcément

\footnotetext{
${ }^{32}$ Fons, J.-P., Meyer, J.-L., (2005). La "flexibilité" dans les fonctions publiques en Angleterre, en Allemagne et en France. Paris, La Documentation française, Perspectives, DGAFP.

${ }^{33}$ Cette question de la dualisation est développée dans : Desmarais C., Jeannot G., Louvel S., Pernot J.-M., Saglio J., Ughetto P.,,, "Gestion des personnels publics évolutions récentes et perspectives", La revue de l'Ires, $n^{\circ}$ 53/1, 2007 p. 111-137.

${ }^{34}$ Jeannot Gilles, "Les fonctionnaires travaillent-ils de plus en plus ? Un double inventaire des recherches sur l'activité des agents publics", Revue française de science politique, vol 58, n 1, p. 123-140.
} 
de bon aloi ${ }^{35}$. Cependant, d'une part la fonction publique d'Etat est loin d'être complètement immobile et d'autre part les blocages à la mobilité sont plus sociologiques que juridiques. Pour passer des principes à la réalité, il faut, là aussi, différencier les agents selon les niveaux hiérarchiques. Pour les cadres, en premier lieu, la mobilité géographique et fonctionnelle est variable, elle peut être très forte, voire trop pour certains cadres des services déconcentrés, mais elle rencontre de nets blocages pour les passages entre ministères voire, comme au ministère des finances, entre directions. Deux raisons non juridiques s'imposent, la première est la différence des niveaux de primes entre ministères qui font que les mieux placés s'accrochent à leur poste. La seconde repose sur un mécanisme d'alliance entre directions et syndicats pour privilégier ceux qui sont déjà là face aux nouveaux venus, qui devront au moins attendre un tour pour être promus ou avoir un poste vraiment intéressant ${ }^{36}$. Pour les agents de catégories $\mathrm{B}$ et $\mathrm{C}$, en second lieu, qui ont des niveaux de salaire et de prime réduits, le principal blocage à la mobilité est lié au coût économique d'un déménagement. Ce blocage est clairement lisible lorsque des individus refusent le bénéfice d'une promotion de grade ou ne se présentent pas à un concours pour ne pas avoir à assumer une mobilité géographique. Dans la mesure où la loi sur la mobilité est muette sur ces questions sociales et économiques, elle à toute chance de venir s'ajouter à d'autres textes qui n'ont pas levé ces difficultés.

Le sujet prend une toute autre tournure avec la révision générale des politiques publiques. En effet, la mise en cause de certains services risque de mettre beaucoup d'agents dans une obligation de mobilité. Cela concerne d'une part des agents de catégories $\mathrm{B}$ et $\mathrm{C}$ peu préparés à la mobilité, mais cela concerne aussi des cadres dont les postes vont être supprimés par la fusion de deux services et qui vont être contraints à la mobilité à un moment où les conditions d'emploi seront difficiles si beaucoup d'entre eux se retrouvent simultanément sur le marché. «La réforme de l'Etat entreprise dans le cadre de la révision générale des politiques publiques implique la suppression d'un grand nombre d'emplois, par la fusion des grandes directions administratives déjà annoncées, et contraint les fonctionnaires concernés à la mobilité. Ce sera le cas, par exemple, lors de la fusion de la direction générale des impôts et de la direction générale de la comptabilité publique, de la carte de la réforme judiciaire, qui prévoit la suppression de plusieurs centaines de tribunaux, et de celle des armées, à travers la réorganisation de leurs fonctions de soutien ${ }^{37}$. Ce sujet, peu évoqué dans le livre blanc, a été au cœur des débats au Sénat sur le projet de loi "mobilité et parcours professionnel dans la fonction publique". Si la mobilité lors de restructuration pourra être encouragée par diverses primes $^{38}$, la mise en mouvement pourra aussi être forcée. L'objet de la discorde portant tout particulièrement sur l'article 7 qui prévoit un statut de "réorientation professionnelle" pour les fonctionnaires dont "l'emploi a été supprimé ou modifié de manière substantielle". "La réorientation peut "prendre fin à l'initiative de l'administration, lorsque le fonctionnaire a refusé successivement trois emplois publics correspondant à son grade et au projet personnalisé d'évolution professionnelle. Dans ce cas il peut être placé en disponibilité d'office, ou, le cas échéant, admis à la retraite ${ }^{39}$ ». Cette mobilité fortement incitée pourrait

\footnotetext{
${ }^{35}$ Dans l'exposé des motifs de la discussion au Sénat du 29 avril 2008, André Santini, cite le cas d'une secrétaire administrative qui aurait acquis des connaissances en matière de subventions agricole et qui ne pourrait exercer cette fonction au ministère de l'agriculture car le corps de «technicien supérieur agricole » ne prévoit pas de détachement en son sein de secrétaire administrative. De tels cas demeurent marginaux.

${ }^{36}$ Thème repéré dans le rapport du Conseil d'Etat, cf. supra.

${ }^{37}$ Hugues Portelli, rapporteur de la commission des lois, Sénat séance du 29 avril, p. 54.

38 Décret $\mathrm{n}^{\circ}$ 2008-367 du 17 avril 2008 instituant un complément indemnitaire en faveur de certains fonctionnaires de l'Etat à l'occasion d'opérations de restructurations. Et l'article 6 de la loi sur la mobilité garantit le maintien du même régime indemnitaire.

${ }^{39}$ La première mouture du texte prévoyait non pas la contrainte de trois offres d'emplois, mais la limite de deux ans. La discussion au Sénat a amené l'amendement « et tenant compte de sa situation de famille ».
} 
aussi être dirigée vers la fonction publique territoriale qui voit ses effectifs régulièrement augmenter alors que celle de l'Etat stagne. La mobilité prend alors un tout autre sens que dans le cas de la gestion routinière des services.

Cette manière d'accompagner fermement un redéploiement des moyens humains n'est pas sans évoquer ce qui s'est passé dans les entreprises publiques qui ont en particulier réaffecté une part importante des agents vers les fonctions de service et de vente. Cette évolution s'est accomplie en combinant plusieurs instruments. En premier lieu, la différenciation du salaire selon le poste occupé a été utilisée pour rendre plus attractives économiquement les fonctions commerciales. En second lieu, les agents dans les services hors du contact avec la clientèle ont été mis en concurrence avec des agents travaillant en intérim et payés à la pièce et les nouveaux recrutements des agents en contacts avec les clients ont été faits sur des contrats de droit privé. Les nombreuses études menées à La poste ont alors mis en évidence un effet de confrontation culturelle notable entre ces agents aux statuts différents et la manière dont ces frottements souvent conflictuels ont conduit à transformer le corps social de ces entreprises ${ }^{40}$.

\section{Conclusion}

Les propositions de réformes ne s'inscrivent donc pas dans un modèle unique. Si certaines propositions comme la prime liée à la performance suivent une affirmation idéologique marquée, d'autres sont plus liées à une certaine continuité de la réforme ou traduisent des contraintes de transcription de règles européennes. Il faut alors s'attacher à saisir les dispositions pratiques qui ont la perspective d'entraîner des effets réels sur la gestion pour appréhender leurs effets combinés.

Si l'on s'efforce alors de rassembler les pièces du puzzle et de mettre en relation les propositions qui semblent pouvoir avoir un impact autre que symbolique, celles-ci s'organisent plus facilement autour d'un transfert de certaines expériences des entreprises publiques autour de la révision générale des politiques publiques qu'autour d'une déclinaison du modèle anglo-saxon du new public management dans le prolongement de la nouvelle loi organique sur les lois de finances. Il est possible de regrouper dans cette perspective les possibilités de mettre en confrontation des agents de statuts différents (intérim, contrat de droit privé avec les fonctionnaires statutaires) ou la proposition de corréler plus fortement le salaire au poste occupé. Le point nodal serait cependant la création d'une obligation de mobilité, qui permet de faire pression sur les agents pour mettre en œuvre, comme l'ont fait ces entreprises, de véritables restructurations.

Cette dernière dimension conduit à poser la question de la différenciation des effets de ces projets de réforme selon les niveaux hiérarchiques. En effet, elle met au centre de la réforme les agents les moins mobiles, c'est-à-dire les plus bas dans la hiérarchie. Et si l'on regarde du côté des plus élevés dans la hiérarchie, d'autres pièces du puzzle se rassemblent : l'instauration de primes au mérite substantielles, la proposition d'un cadre d'emploi spécifique pour les fonctions de direction qui permette de définir plus librement les modes de rémunérations et l'ouverture de ces emplois à des personnes issues du privé ou, de manière plus générale, la capacité des "grand corps" de s'être mis à l'écart des réformes. Ainsi les effets des réformes en cours devront être tout autant évaluées à l'aune des répartitions entre niveaux de

\footnotetext{
${ }^{40}$ Aubert N., De Gaulejac V., modernisation et résistance dans un grand établissement de courrier, les avatars d'un système perdant perdant", rapport effectué pour la compte de la mission de la recherche de La poste, 1998. Piotet $\mathrm{F}$ et al, Les conseillers financiers de La poste, synthèse des résultats, mission de la recherche de La poste, 1998.
} 
fonctionnaires, des gagnants et des perdants de ces réformes, qu'à celle des évolutions des principes et valeurs de la fonction publique. 